Methods: A population-based case-control study using incident cases of RA was performed in Sweden, and the study population in this report was restricted to include never smokers (589 cases, 1764 controls). The incidence of RA among never smokers who had been exposed to passive smoking was compared with that of never smokers who had never been exposed, by calculating the odds ratio with a $95 \%$ confidence interval employing logistic regression.

Results: No association was observed between exposure to passive smoking and RA risk (OR 1.0, 95\% Cl 0.8-1.2 for ACPA positive RA, and OR 0.9, 95\% Cl $0.7-1.2$ for ACPA negative RA). No suggestion of a trend between duration of passive smoking and RA risk was observed.

Conclusions: No association was observed between exposure to passive smoking and RA risk, which may be explained by a threshold below which no association between smoke exposure and RA occurs.

Acknowledgements: The EIRA study was supported by grants from the Swedish Medical Research Council, the Swedish Council for Health, Working life and Welfare, King Gustaf V:s 80-year foundation, the Swedish Rheumatism Foundation, Stockholm County Council, the insurance company AFA, the EUsupported AutoCure and BeTheCure projects, and from the COMBINE (Controlling chronic inflammatory diseases with combined efforts) project.

Disclosure of Interest: None declared

DOI: 10.1136/annrheumdis-2017-eular.3389

\section{SAT0722-HPR FAMILIAL RISKS OF RHEUMATOID ARTHRITIS: EVIDENCE FROM THE MALAYSIAN EPIDEMIOLOGICAL INVESTIGATION OF RHEUMATOID ARTHRITIS CASE-CONTROL STUDY}

C.L. Too ${ }^{1,2}$, L.K. $\operatorname{Tan}^{3}$, A.F. Nurul Aain ${ }^{3}$, S. Salsabil ${ }^{3}$, H. Heselynn ${ }^{4}$, S. Wahinuddin ${ }^{5}$, I.S. Lau ${ }^{6}$, S.C. Gun ${ }^{7}$, S. Nor-Shuhaila ${ }^{4}$, M. Eashwary ${ }^{4}$, M.S. Mohd-Shahrir ${ }^{8}$, M.-M. Ainon ${ }^{9}$, R. Azmillah ${ }^{6}$, O. Muhaini ${ }^{10}$

C. Bengtsson ${ }^{11}$, L. Padyukov ${ }^{2}$, L. Alfredsson ${ }^{11}$, L. Klareskog ${ }^{2}$, M. Shahnaz ${ }^{12}$. ${ }^{1}$ Allergy and Immunology Research Center, Institute for Medical Research, Kuala Lumpur, Malaysia; ${ }^{2}$ Department of Medicine. Rheumatology Unit, Karolinska Institutet and Karolinska University Hospital, Stockholm, Sweden; ${ }^{3}$ Institute for Medical Research, Kuala Lumpur; ${ }^{4}$ Department of Medicine, Putrajaya Hospital, Putrajaya; ${ }^{5}$ Faculty of Medicine, Universiti Kuala Lumpur Royal College of Medicine Perak, Ipoh; ${ }^{6}$ Department of Medicine, Selayang Hospital, Selayang; ${ }^{7}$ Department of Medicine, Hospital Tunku Ja'afar Seremban, Seremban; ${ }^{8}$ Faculty of Medicine, Universiti Kebangsaan Malaysia Medical Center, Kuala Lumpur; ${ }^{9}$ Department of Medicine, Tengku Ampuan Afzan Hospital, Kuantan, Pahang; ${ }^{10}$ Department of Medicine, Hospital Raja Perempuan Bainun, Ipoh, Perak, Malaysia: ${ }^{11}$ Institute of Environmental Medicine, Karolinska Institutet, Stockholm, Sweden; ${ }^{12}$ Ministry of Health. Level 12th, Block E7, Parcel E, Federal Government Administrative Center, Putrajaya, Malaysia

Background: Family history of rheumatoid arthritis (RA) is a surrogate for an individual's genetic and partly environmental risk of developing RA. It is assessed daily in clinical practice and its magnitude and pattern of distribution may provide information on the RA etiology.

Objectives: We investigated the association between family history of RA and the risk of anti-citrullinated peptide antibody (ACPA)-positive and ACPA-negative RA in the Malaysian population.

Methods: Data from the Malaysian Epidemiological Investigation of Rheumatoid Arthritis (MyEIRA) population-based case-control study involving 1,055 early RA cases and 1,055 age, sex and residential area-matched controls were analyzed. Information from interview-reported family history of RA or rheumatic stiff back among first degree relatives was used to estimate the risk of developing ACPApositive and ACPA negative RA. The odds ratio (OR) with $95 \%$ confidence interval (Cl) was calculated.

Results: In this study, $64 \%$ of the RA patients were ACPA-positive and $40 \%$ of the overall RA carried HLA-DRB1 shared epitope (SE) alleles. Family history of RA was significantly associated with an increased risk of developing RA in the Malaysian population (RA versus controls, $17.0 \%$ vs. $7.7 \%$, OR $2.4,95 \%$ $\mathrm{Cl} 1.8-3.2, \mathrm{p}<0.0001)$. The association between positive family history and risk of RA was uniformly observed for the ACPA-positive RA (OR $2.5,95 \% \mathrm{Cl}$ 1.8-3.3, $\mathrm{p}<0.0001$ ) and ACPA-negative RA (OR 2.3, 95\% Cl 1.6-3.2, $\mathrm{p}<0.0001$ ) subsets, respectively. A dramatically increased risk for ACPA-positive RA was seen in individuals who both were having positive family history of RA and carried HLA-DRB1 SE alleles (OR 14.7, 95\% CI 7.7-27.8). We also observed a lesser risk magnitude in the ACPA-negative RA patients (OR 5.7, 95\% Cl 2.7-11.9). Conclusions: Our data demonstrate that family history of RA remains an important clinical risk factor for RA. In addition, positive family history of RA was associated with an increased risk of developing both the ACPA-positive and ACPA-negative RA in the Malaysian population, suggesting that the two RA subsets are similar in genetic risk factors that overlap with these diseases.

References:

[1] Frisell T, Saevarsdottir S, Askling J. Family history of rheumatoid arthritis: an old concept with new developments. Nat Rev Rheumatol. 2016 Jun;12(6):335-43.

[2] Frisell T, Hellgren K, Alfredsson L, Raychaudhuri S, Klareskog L, Askling $\mathrm{J}$. Familial aggregation of arthritis-related diseases in seropositive and seronegative rheumatoid arthritis: a register-based case-control study in Sweden. Ann Rheum Dis. 2016 Jan;75(1):183-9.

Disclosure of Interest: None declared
DOI: 10.1136/annrheumdis-2017-eular.4714

\section{SATURDAY, 17 JUNE 2017 HPR patients' perspectives, functioning and health (descriptive: qualitative or quantitative)}

\section{SAT0723-HPR GENDER DIFFERENCES IN COPING STRATEGIES AND ILLNESS ACCEPTANCE IN PATIENTS WITH INFLAMMATORY ARTHRITIS - A NATIONWIDE CROSS-SECTIONAL STUDY}

B.A. Esbensen ${ }^{1,2}$, C. Flurey ${ }^{3}$, K.V. Jensen ${ }^{1}$, L. Andersen ${ }^{1}$, N.M. Hammer ${ }^{1}$. ${ }^{1}$ Rigshospitalet - Glostrup, COPECARE, Centre for Rheumatology and Spine Diseases \& Research Unit, Glostrup; ${ }^{2}$ Department of Clinical Medicine, Faculty of Health and Medical, University of Copenhagen, Copenhagen, Denmark; ${ }^{3}$ Rheumatology Unit, Bristol Royal Infirmary, West England, Bristol, United Kingdom

Background: There is an increasing focus on how patients with inflammatory arthritis (IA) manage living with arthritis. There are a preponderance of women with RA $(70 \%)$, thus previous research has overall focused on female patients and their management. Research in other long term conditions suggests men need their own health strategy ${ }^{1}$, thus it is important to investigate whether there are gender differences in coping strategies and illness acceptance within chronic IA. Objectives: To explore gender differences in IA as reflected by coping strategies and illness acceptance. Furthermore, to identify factors associated with high degree of illness acceptance.

Methods: The study was conducted as a nationwide cross-sectional study using online survey during 2016. Patients $>18$ with rheumatoid arthritis (RA), psoriatic arthritis (PsA) and axial spondyloarthritis (axSpA) were invited to contribute through: The Danish Rheumatism Organization, local arthritis networks, diagnosis networks, and rheumatology departments across the country. The selfreport online questionnaire comprised Socio-demographics, Diagnosis, Symptoms (pain, fatigue, global), Medications, Disease Activity, Functional Status, Coping (i.e. confrontation, avoidance and acceptance-resignation) and IIIness Acceptance. As recommended by EULAR 2 patients with RA (KVJ \& LA; male and female, respectively), were included as equal research partners in all phases of the study. Descriptive statistics were applied to explore gender differences, and logistic regression analyses were performed to test for factors associated with illness acceptance.

Results: In total, 664 (85\% women) were included in the study; RA 53\%, PsA $27 \%$ and axSpA $20 \%$. More men $(40 \%)$ than women $(30 \%)$ were treated with biological DMARDS $(p=0.048)$. No significant gender differences were found in disease activity, symptoms and functional status. Overall, the total sample had high degree of illness acceptance and no significant difference was found between males and females. Regarding illness coping, women with IA tend to use avoidance as a coping strategy significantly more than men $(\mathrm{p}=0.015)$. In the final multivariable regression model, higher education $(\mathrm{OR}=1.46 ; 1.02-2.11)$, longer time diagnosed (OR=1.21 per 1-yr. increase; 1.01.-1.05), lower physical disability $(\mathrm{OR}=0.76 ; 0.69-0.85)$, better coping with fatigue $(\mathrm{OR}=1.13 ; 1.05-1.22)$, less avoidance $(\mathrm{OR}=0.93 ; 0.87-0.99)$ and acceptance-resignation $(\mathrm{OR}=0.62$; $0.62-0.75$ ) as coping strategies were significantly associated with high degree of illness acceptance.

Conclusions: No significant differences were found in illness acceptance among women and men with IA. However, women tended to use avoidance as a coping strategy more than men. High levels of illness acceptance may be explained by high education, longer disease duration, and better physical function, better coping of fatigue and less use of passive coping strategies.

[1] White A, McKee M, Richardson N, et al. Europe's men need their own health strategy. BMJ 2011;343:d7397.

Disclosure of Interest: None declared

DOI: 10.1136/annrheumdis-2017-eular.5027

\section{SAT0724-HPR GOTHENBURG NURSE LED TIGHT CONTROL STUDY - GOTNET. PATIENTS' EXPERIENCES OF NURSE-LED CARE AND TIGHT CONTROL. A QUALITATIVE STUDY OF PATIENTS WITH RHEUMATOID ARTHRITIS}

U. Bergsten ${ }^{1,2}$, A.-S. Sjö ${ }^{1} .{ }^{1}$ Rheumatology Department, Sahlgrenska University Hospital, Gothenburg; ${ }^{2}$ Department of Research, Development and Education, Region Halland, Varberg, Sweden

Background: Rheumatoid arthritis (RA) is a chronic inflammatory illness, using both pharmacological and non-pharmacological therapies. Some patients experience good treatment, knowledge about their illness and situation. For other patients, it might look different depending on how much they know about their illness. Earlier research has primary focused on patients in remission. In order to strengthen and support patients with RA that are not feeling well, to give them more knowledge about their situation, one strategy could be that they have access to a nurse-led rheumatology clinic with person-centered care (PCC). 
Objectives: The aim of this study was to describe patients' experiences of nurse-led care tight control.

Methods: Qualitative design was used in order to receive deeper understanding of the phenomenon. Interviews were conducted with 12 informants (with strategic sample) that participated in the intervention group of a randomized controlled trial exploring the effect of patient centered care, the Got-Net study. The interviews were analyzed using phenomenography approach.

Results: The informants' experiences of nurse-led care tight control emerged in three categories. The three categories describe the process, the relationship between the concepts. The first step describes the Meeting of competence, the next step that the informant experienced was Relationship building and the last step to have Made a personal journey.

Conclusions: Patients with RA who got the opportunity of nurse-led care and tight control became strengthen, got extended knowledge about their illness. Patients have been treated on their own level, knowledge and needs, by using a person-centered approach.

Disclosure of Interest: None declared

DOI: 10.1136/annrheumdis-2017-eular.4675

\section{SATURDAY, 17 JUNE 2017}

\section{HPR interventions (educational, physical, social and psychological)}

\section{SAT0725-HPR GOTHENBURG NURSE LED TIGHT CONTROL STUDY - GOTNET. A STUDY COMPARING "CARE AS USUAL" WITH NURSE LED CLINIC, TIGHT CONTROL AND PERSON CENTRED CARE IN PATIENTS WITH RHEUMATOID ARTHRITIS (RA) AND WITH MODERATE/HIGH DISEASE ACTIVITY}

U. Bergsten ${ }^{1,2}$, K. Almehed ${ }^{2}$, L. Jacobsson ${ }^{3} .{ }^{1}$ Department of Research, Development and Education, Region Halland, Varberg: ${ }^{2}$ Rheumatology Department, Sahlgrenska University Hospital, Gothenburg; ${ }^{3}$ Department of Rheumatology a Inflammation Research, Institute of Medicine, Gothenborg, Sweden

Background: The treatment guidelines of EULAR recommend treatment according to "tight control" (TC), clear treatment goals (Treat-to-Target, T2T) and patient involvement. Despite this, the care for patients with RA needs to be improved as demonstrated by the fact that as many as that $45 \%$ of patients with RA in Sweden (according to the Swedish Rheumatology Quality Register in 2015) do not reach the level of low disease activity. One way to overcome this could be nurse led clinics of RA patients, which for patients in remission have shown positive effects on patient's pain, knowledge and self-efficacy. We hypothesized that a nurse-led clinic based on the above principles of TC, T2T and Person-centered care would result in better outcome compared to care as usual.

Objectives: The aim of the study was to compare the effect of such nurse-led clinic with frequent visits and person-centered care with regular care of patients with established RA and a moderate to high disease activity.

Methods: This was a randomized, controlled, blinded assessor study that aimed to compare the effect of nurse-led clinic with regular care of patients with the established RA. Inclusion criteria were; duration of RA $>2$ yrs., DAS28 $>3.8$, age $<85$ yrs. and not having experience with all available csDMARDs and bDMARDS. Patient with any severe current comorbidity were excluded. Intervention Group (IG): intervention included two elements; 1) disease activity control every 6th week, with clear treatment goals (DAS28 <2.6)and escalation of pharmacological therapy according to existing Swedish guidelines, 2) a written person-centered care health plan with shared goal setting and decision making between patient and nurse. Control Group (CG): Patients in the control group were followed by a physician once every 3 to 6 month. Evaluation was done at w 26 both per protocol (shown) and according to intention to treat (ITT) with LOCF. Primary outcome was change in DAS28 at week 26 and secondary outcome the proportions of $\mathrm{good} / \mathrm{moderate}$ EULAR response. Pre study power calculations estimated group sizes to 60 patients in each group. Due to difficulties in the recruiting process the study was stopped preterm

Results: The study included 70 patients of which (IG: 36 and CG: 34) with similar baseline characteristics as a result of the randomization. Sixty-two patients completed the study at w 26 (IG: 29 and CG: 33). Both groups improved significantly in DAS 28 (Table 1). The change in DAS 28 was not significantly different between groups, although the numerical improvement was larger in the IG compared to CG (Table 1). Furthermore, significantly more patients in the IG improved according to EULAR Good or Moderate response (Table 1). Results were similar when analyzing according to ITT with LOCF.

Table 1. Primary outcome (delta DAS 28) and secondary outcome (good/moderate EULAR response)

\begin{tabular}{lccc}
\hline & IG & CG & Difference $(95 \% \mathrm{Cl})$ \\
\hline DDAS 28 (95\% Cl) & $1.5(-2.0,-1.0)$ & $1.1(-1.6,-0.6)$ & $-0.43(-1.13,0.27)$ \\
EULAR Good/Moderate (\%) & $22 / 29(76 \%)$ & $16 / 33(48 \%)$ & $27 \%(3-51 \%)$
\end{tabular}

Conclusions: This controlled trial suggest that a nurse-led clinic for RA patients with moderate/high disease activity may be effective. Although not shown, it likely to be cost-effective and possible to implement in clinical care.

Disclosure of Interest: None declared

DOI: 10.1136/annrheumdis-2017-eular.2659

\section{SATURDAY, 17 JUNE 2017}

\section{HPR patients' perspectives, functioning and health (descriptive: qualitative or quantitative)}

\section{SAT0726-HPR FUNCTIONAL IMPAIRMENT IN PATIENTS WITH RA IN AN EIGHT YEAR PERSPECTIVE}

A. Bremander $^{1,2,3}, \mathrm{~K}$. Forslind ${ }^{4,5}, \mathrm{~K}$. Eberhardt ${ }^{1}, \mathrm{M}$. Andersson ${ }^{1,3}$. ${ }^{1}$ Department of Clinical Sciences, Section of Rheumatology, Lund University, Lund; ${ }^{2}$ School of Business, Engineering and Science, Halmstad University; ${ }^{3}$ Spenshult Research and Devlopment Centre, Halmstad; ${ }^{4}$ Department of Clinical Sciences, Section of Rheumatology, Lund University, Lund and Helsingborg; ${ }^{5}$ Department of Research and Education, Helsingborg's hospital, Helsingborg, Sweden

Background: In people with Rheumatoid arthritis (RA) impaired physical functioning is an acute as well as long term effect of the disease. Observational performance tests reflecting range of motion in upper as well as in lower extremities should be easy to perform in the clinic as well as in research as a complement to self-reported measures of physical functioning. The Signal Of Functional Impairment $(\mathrm{SOFI})^{1}$ is a performance test which so far has been applied only in Sweden but commonly used in the clinic and in long term follow-up clinical studies. Objectives: The aim was to study performance-based function assessed with SOFI over 8 years and, secondly, to study which items included in SOFI that were associated with change in functioning over time.

Methods: An inception cohort of 1052 patients with early RA, from the BARFOTstudy, recruited 1992-2006 was investigated, mean (SD) age was 54 years (14), $70 \%$ were women. The patients were followed by a structured protocol at baseline, 3 and 6 months and at 1,2,5, and 8 years. SOFI consists of 3 parts measuring hand, arm (upper), and leg (lower) function (1). Hand function is tested by 4 movements; cylinder grip $(\mathrm{H} 1)$, pen grip $(\mathrm{H} 2)$, pincer grip $(\mathrm{H} 3)$ and opposition of the thumb ( $\mathrm{H} 4)$. Arm function is assessed by 3 movements; hand behind the head and the ability to touch the cervical spine processes with fingers (A1), elbow supination (A2) and elbow extension (A3). Leg function is tested by 4 movements; the ability to touch the opposite knee with the heel while sitting (L1), knee extension in supine position (L2), dorsiflexion of the foot standing on a balance board (L3), and the ability to stand on tip toes without shoes (L4). An assessor scores the patient's ability to perform the different tests on an ordinal scale $(0=$ normal, $1=$ partly impaired and $2=$ unable to perform). The range of SOFI scores is $0-44$ (best to worst).

Results: At baseline the mean (SD) SOFI was 7.2 (5.8), and at 1 year follow-up the improvement was $2.75(5.65), p<0.001$. From 1 year to 8 year follow-up the deterioration was 1.5 (4.6), $p<0.001$. When studying hand, upper and lower function separately, the pen grip and the ability to stand on tip toes improves most during the first year. From 1 to 8 year the pincer grip and the ability to stand on tip toes are the items that deteriorate most (Figure). Assessment of the pen grip, the pincer grip and the ability to stand on tip toes explain $58 \%$ to $70 \%$ of the SOFI score over time, with the highest rate at $5(65 \%)$ and 8 years follow-up $(70 \%)$.
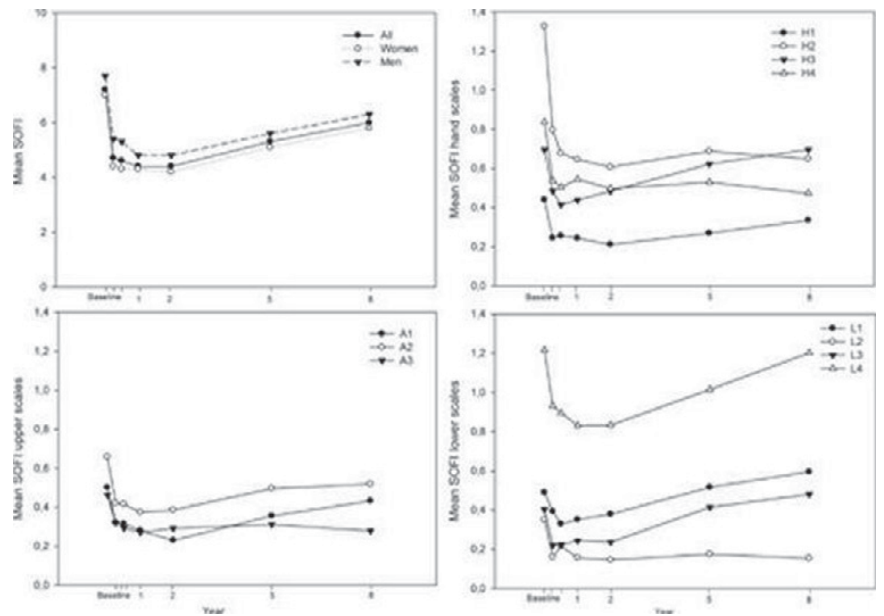

Conclusions: Functioning as assessed by SOFI improved during the first year in patients with early RA and then deteriorated slowly. Over a longer period, pincer grip and the ability to stand on tip toes seemed to be the two most important items to measure when assessing functional impairment over time.

References:

[1] Kerstin B. Eberhardt, B. Svensson and U. Moritz. Functional assessment of early rheumatoid arthritis British Journal of Rheumatology 1988;27:364-371. 